\title{
The Effects of Different Monochromatic Colors on Oxidant Antioxidant Balance in Broilers
}

\author{
๑ Nilay SEYIDOGLU ${ }^{1}, *$, ๑ Deniz BELENLI ${ }^{2}$, \\ ๑ Oguz MERHAN ${ }^{3}$, $\odot$ Kadir BOZUKLUHAN ${ }^{4}$
}

\begin{abstract}
1Department of Physiology, Faculty of Veterinary Medicine, University of Tekirdag Namik Kemal, TR-59030 Tekirdag TURKEY 2 Department of Biochemistry, Faculty of Veterinary Medicine, University of Tekirdag Namik Kemal, TR-59030 Tekirdag TURKEY 3 Department of Biochemistry, Faculty of Veterinary Medicine, University of Kafkas, TR-36100 Kars TURKEY 4 Kars School of Higher Vocational Education, University of Kafkas, TR-36100 Kars TURKEY
\end{abstract}

Received 2019-11-01 Accepted 2020-02-13

\begin{abstract}
Poultry management is associated with light due to positive effects of photoreceptors on animal's growth, immunity and homeostasis. Colors influence the efficiency of poultry performance and behavior. Light-emitting diode (LED) can use for providing monochromatic light source. In this trial, two hundred Ross 308 broiler chicks were studied in four different light groups as follows; White (Control), Red (RL), green (GL) and blue (BL) during 42 trial days. At the end of the study, the serum Superoxide dismutase (SOD), Catalase (CAT), Glutathione Peroxidase (GPx) and malondialdehyde (MDA) enzyme activities were measured to evaluate the oxidant-antioxidant balance. The oxidant marker MDA was found the highest value in group RL (p:0.0001; 9.24 \pm 0.33 ). On the other hand, in group GL, all antioxidant parameters were found the highest value $(\mathrm{p}<0.05)$. Also, SOD and GPx values were increased in group GL (SOD: 150.61 \pm 9.14 ; GPx: 186.96 \pm 10.50$)$ than BL (SOD: 139.29 \pm 5.19 ; GPx: 160.02 \pm 3.15$)$ statistically $(\mathrm{p}<0.05)$. The results showed that red light may be a stressor, and on the contrary, green light may be a positive monochromatic color for growth, oxidant-antioxidant balance and homeostasis for broilers.
\end{abstract}

Key words: poultry, green light, antioxidant enzymes, MDA

\section{Introduction}

The light is converted to impulses to brain and effects on several physiological mechanisms ${ }^{1}$. Also, types of photoreceptors in the eye have a complex process for visual system. However there are some differences between human and animal vision. The human eye detects a narrow range between 400-700 $\mathrm{nm}$ wavelengths on the scale in the electromagnetic spectrum. On the other hand, chickens can perceive smaller wavelengths $(350 \mathrm{~nm})$ than humans as superior. Thus, they can sense the wavelengths of light in the wider spectrum. ${ }^{2,3}$ Chickens have seven photoreceptors in their eyes to separate the light colors. Besides that, they have five light receptors in their eyes which get an ability to see many colors to them. ${ }^{4}$ According to these structures, lightening is regarded an environmental stress factor for chicken's physiology and poultry management. ${ }^{5}$ Many mechanisms are influenced by colors of light, especially growth performance, reproductivity, egg production and quality. ${ }^{6,7}$

Color is an important aspect of light due to its effect on several physiological mechanism and behavior in poultry. The artificial colors used for poultry management are named white, red, green and blue. Red light gives animal more activity such as walking, flapping, aggression and 
cannibalism. ${ }^{78}$ Green and blue lights are called tranquilizer of the study. The chicks of one day age were placed in colors, and stimulate drinking, bathing, sleeping, sitting 4 deep cages in the trial room isolated from daylight which have positive impact on animal growth, reproduc- and a black screen was drawn between each tivity and immunity. ${ }^{7,910}$ It was reported that both green and blue lights reduce negative effects of stress and maintains the immunity. ${ }^{11}$ Also, increase of the antioxidant enzyme activities by blue light in poultry was determined by some researchers. ${ }^{12,13}$

The oxidant antioxidant balance in healthy status can be explained with superiority of antioxidant capacity. In animals, stressful stiuations such as long lightening, light colors, crowded, nutrition and etc. cause a damage in this balance, and oxidation is occured. ${ }^{14}$ Nevertheless, several pathological conditions could be existed in growth, immunity, reproductivity and animal products. ${ }^{15-17}$ The first defense systems responded the cellular homeostasis are antioxidant enzymes. ${ }^{18}$ Malondialdehyde (MDA) is known as the most important molecule for oxidative status in tissue or cells. Belong to oxidative status, antioxidants are released to fight for homeostasis, however if they're insufficient, exogenous antioxidants could be given to regulate the oxidant antioxidant balance. ${ }^{19}$ The best natural antioxidants in organism are carotenoids, glutathione (GSH), glutathione peroxidase (GPx), superoxidase dismutase (SOD) and catalase (CAT) which also act as a free radical scavenger by eliminate the lipid peroxidation and oxidative damages. ${ }^{20,21}$

Light-emitting diode (LED) is a popular monochromatic light source commercially. The advantage of LED lamps is single intensity of wavelength which has a narrow half band output. ${ }^{22}$ LED light spectra impacts photoperiodic answer of birds. This effect of LED light influences the broiler's growth, behavior, immune response, physiological and biochemical alterations. ${ }^{23,24}$ According to these literatures, it can be said that light management is important for improvement of poultry productivity and welfare of animals. In this trial, we aimed to determine the effects of three monochromatic LED lamps in colors red, green and blue on oxidant and antioxidant enzyme activities in broilers.

\section{Material Methods}

\section{Birds and Housing}

The experiment was carried out in chicken coops in the Animal Husbandry Facilities of the Agriculture Faculty, Tekirdag Namik Kemal University. The study was carried out with the permission of University Animal Experimentation Local Ethics Committee (Approval number:

T2017-7-5). A total of 200 Ross 308 broiler chicks were obtained from a parent flock randomly mating for animal material compartment. The birds were housed d 0 in separate rooms.

Groups were divided into 4 as follows respectively: White light (Control, 400-760 nm), Red light group (RL, 635-700 nm wavelength), Blue light group (BL, 450-490 nm wavelength) and Green light group (GL, 490-560 nm wavelength). Each trial group was randomly allocated 50 animals. LED lighting fixtures were mounted on each cage and adjusted so that there was no reflection on the other cages. The LED lamps were placed $10 \mathrm{~cm}$ above the head of broilers. The light was arranged period of 23 hour (23L:1D). Chicks were fed with 24\% HP and $2900 \mathrm{kcal} / \mathrm{kg}$ ME powdered feed throughout the trial and ad libitum access to feed and water during 42 trial days.

\section{Blood samples and Antioxidant Status}

Birds were slaughtered at the end of the trial and the blood samples were taken from jugular vein at the slaughter day. 10 of each group totally 40 samples were collected into anticoagulant blood tubes. Plasma was obtained by centrifugation at $3000 \mathrm{~g}$ for 10 minutes and stored at $-20{ }^{\circ} \mathrm{C}$ until analyses day. Antioxidant enzymes in plasma such as Superoxide dismutase (SOD), Catalase (CAT) and Glutathione Peroxidase (GPx) enzyme activities were measured with commercial kits (Cayman Chemical Company, USA) by microplate reader and malondialdehyde (MDA) levels were measured according to the colorimetric method by Yoshoiko et al. ${ }^{25}$ with spectrophotometer.

\section{Statistical analysis}

All the values were grouped and calculated as means \pm standard errors. Statistical analyses performed with SPSS (Version 20.0). All parameters were examined by One-way for the difference between groups and considered significant at $\mathrm{p}<0.05$. If the difference between groups was provided to be significant $(\mathrm{p}<0.05)$, Tukey's test used. ${ }^{26}$ Nevertheless, Kruskal Wallis was used for analyzed the differences be-tween means in nonhomogenous groups, and following Mann Whitney $U$ test applied between groups one by one. ${ }^{27}$

\section{Results}

In the study, the oxidant marker, MDA, was determined for stressful condition. The MDA value was increased in group red light (RL) compared to group Control (p:0,0001; 5.48 \pm 0.12 and 9.24 \pm 0.33 , group 

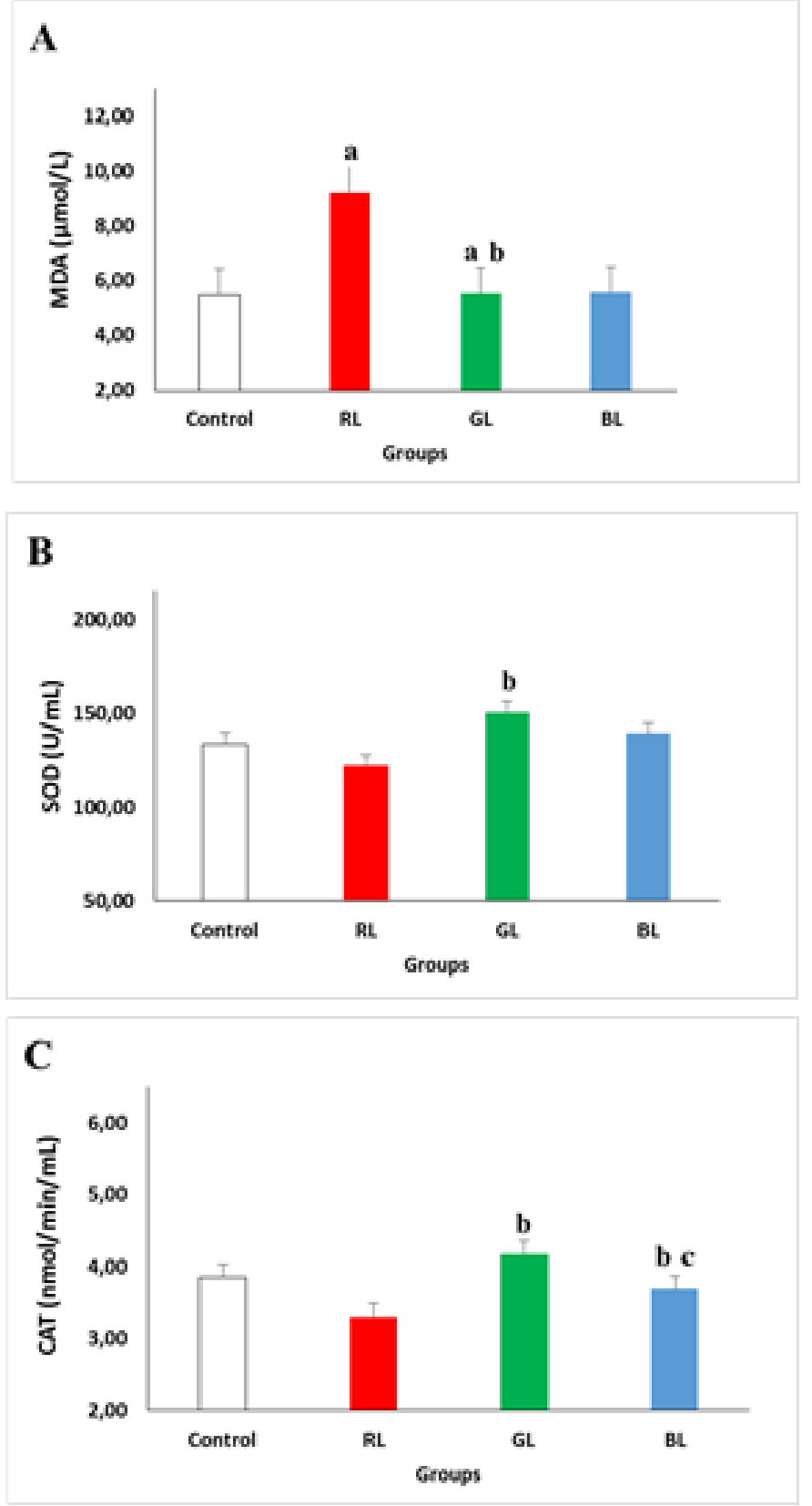

D

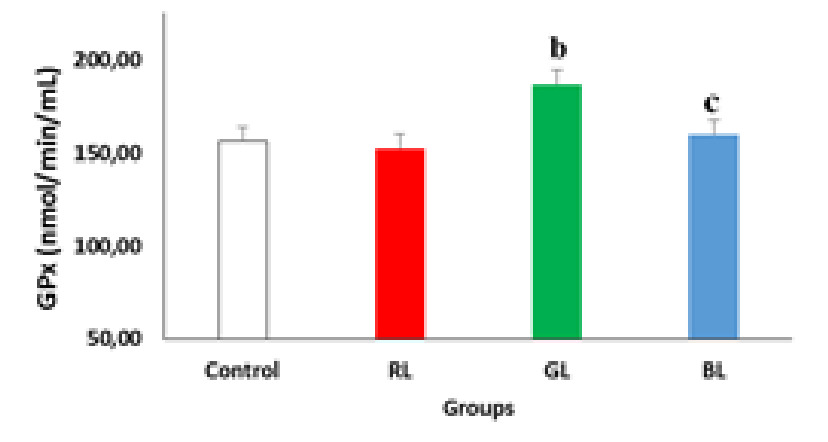

Figure 1: The effect of monochromatic light differences on the change of serum oxidant and antioxidant values. A) Malondialdehyde (MDA) enzyme value; B) Superoxide dismutase (SOD) enzyme value; C) Catalase (CAT) enzyme value ; D) Glutathione Peroxidase (GPx) enzyme value.

Groups: Control, RL: red light, GL: green light, BL: blue light, respectively.

* $\mathrm{p}<0.05, \mathrm{RL}, \mathrm{GL}$ and BL versus $\mathrm{C}$ group

$\neq \mathrm{p}<0.05, \mathrm{GL}$ and $\mathrm{BL}$ versus RL group

$+\mathrm{p}<0.05$, GL versus BL group were found higher in groups GL (SOD, CAT and GPx respectively; $150.61 \pm 9.14,4.18 \pm 0.07$ and $186.96 \pm 10.50$ ) and BL (SOD, CAT and GPx respectively; 139.29 \pm 5.19 , $3.69 \pm 0.08$ and $160.02 \pm 3.15)$ compared to Control group (SOD, CAT and GPx respectively; 133.68 $\pm 8.49,3.86 \pm 0.18$ and $156.14 \pm 5.67$ ) statistically (Fig1b-1c-1d; $<<0.05)$. On the other hand, between group Green and Blue, differences were found in SOD and GPx statistically (SOD and GPx $p$ values respectively, 0,007 and 0,038).

\section{Discussion and Conclusion}

In poultry management, lightening procedure is important due to the chickens' sensitivity to light color or lightening periods. Light has a stimulating effect on photoreceptors that results in release of hormones for development of stress and aggression. ${ }^{28,29}$ To evaluate the stress which from oxidant and antioxidant capacity is necessary to define. Organisms have specific molecules for determining this oxidation cellularly such as MDA, GSH, SOD, GPx and CAT etc. There is a balance between these oxidant and antioxidant molecules for homeostasis. Although stress gives an increase in oxidants, oxidation lead to decrease in antioxidants. However, to generate the metabolism in that situation, antioxidants are produced and oxidative damage is repaired. ${ }^{30,31}$

Our results showed that oxidant and antioxidant balance of broiler were affected by LED light colors (Figure 1). Animals exposed under red light had the higher MDA value, which can be a stress marker, compared to group control $(\mathrm{p}<0.0001)$ although there was no differences were found among groups $\mathrm{GL}$ and $\mathrm{BL}(\mathrm{p}>0.05)$. This result may be due to the red light improvement effect on cellular oxidative activity. Similarly, it was reported that red light had a stress effect such as cannibalism in hens. ${ }^{32}$

Several studies suggested that monochromatic colors, green and blue have positive effect on growth and welfare against to stress especially in poultry management. ${ }^{7,10} \mathrm{In}$ the present study, the antioxidant enzymes values were increased in groups GL and BL compared to group Control statistically $(\mathrm{p}<0.05)$. Animals reared under green light (group GL) had the highest SOD (150.61 \pm 9.14$)$, CAT $(4.18 \pm 0.17)$ and GPx $(186.96 \pm 10.50)$ values compared to group Control and BL (Fig 1b-1c-1d; $\mathrm{p}<0.05)$. These increases of antioxidative enzymes may be explained by oxidative defense mechanism which occurs with cellular protein expression. Abdo et al. ${ }^{13}$ determined that blue light could maintain the mRNA expression, and thereby proteins known as SOD, CAT and GPx1, which are important in oxidative antioxidative balance were increased. There are some reports about the efficiency of green and blue light on bird's growth. ${ }^{10,33}$ Those studies, it was indicated 
that although blue light induces the growth in old age of birds, green light stimules the growth in youngers. Woodard et al. ${ }^{34}$ also reported that green and blue light has the positive effect in Japanese quails. Monochromatic colors have been important for poultry management, especially green and blue. The obtained results suggest that either green or blue light has the best result for antioxidative balance of organism and maintain welfare. Thereby, green and blue light colors may be the sources for lightening process in poultry management. Furthermore, it can be said that green and blue light might be the best colors replace to others in broiler production.

\section{References}

1. Er D, Wang Z, Cao J, et al. Effect of monochromatic light on the egg quality of laying hens. The Journal of Applied Poultry Research. 2007;16(4):605-612.

2. North M, Bell DD. Lighting management in: Chicken Production Manual.1990;407-431.

3. Prescott, NB, Wathes CM. Spectral sensitivity of the domestic fowl (Gallus g. Domesticus). Br Poult Sci. 1999;40: 332-339.

4. Hart NS. Variations in cone photoreceptor abundance and the visual ecology of birds. Journal of Comparative Physiology A. 2001;187: 685-97.

5. Olanrewaju HA, Thaxton JP, Dozier WA, et al. A review of lighting programmes for broiler production. Int J Poult Sci. 2006; 5(4):301-308.

6. Nicholls TJ, Goldsmith AR, Dawson A. Photorefractoriness in birds and comparison with mammals. Physiological Reviews. 1998;68, 133-176.

7. Hesham MH, Shereen A-H El, Enas SN. Impact of different light colours in behavior, welfare parameters and growth performance of Fayoumi broiler chickens strain. J Hell Vet Med Soc. 2018; 69(2):951-958.

8. Khaliq T, Khan AA, Dar PA, et al. Behavioral study of broilers reared under different colours of light in the evening hours. J Entomol Zool Stud. 2018; 6(4):16241627.

9. Rozenboim I, Robinzon B, Rosenstrauch A. Effect of light source and regimen on growing broilers. Br Poult Sci. 1999; 40(4):452-457

10. Rozenboim I, Chaiseha Y, Rosenstrauch A. The effect of a green and blue monochromatic light combination on broiler growth and development. Poult Sci. 2004; 83(5):842-845.

11. Xie D, Wang ZX, Dong YL et al. 2008. Effects of monochromatic light on immune response of broilers. Poult Sci. 2008; 87(8): 1535-1539.
12. Ke YY, Liu WJ, Wang ZX, et al. Effects of monochromatic light on quality properties and antioxidation of meat in broilers. Poultry Science. 2011;90(11): 26322637.

13. Abdo SE, El-Kassas S, El-Nahas AF, et al. Modulatory Effect of Monochromatic Blue Light on Heat Stress Response in Commercial Broilers. Oxidative Medicine and Cellular Longevity. 2017; 1-13.

14. Tanno S, Yamamoto K, Kurata Y, Adachi M, Inoue Y, Otani N, Mishima M, Yamamoto Y, Kuwabara M, Ogino K, Miake J, Ninomiya H, Shirayoshi Y, Okada F, Yamamoto K, Hisatome I. Protective Effects of Topiroxostat on an Ischemia-Reperfusion Model of Rat Hearts. Circulation Journal. 2018; 82:1101-1111.

15. Abdel-Rahman HA, Shawky SM, Ouda H, Nafeaa AA, Orabi SH. Effect of two probiotics and bioflavonoids supplementation to the broilers diet and drinking water on the growth performance and hepatic antioxidant parameters. Global Veterinaria. 2013; 10:734-741.

16. Fellenberg A, Speisky HC. Antioxidants: their effects on broiler oxidative stress and its meat oxidative stability. World's Poultry Science Journal. 2006; 62(1):53-70.

17. Iskender H, Yenice G, Dokumacioglu E, Kaynar O, Hayirli A, Kaya A. The Effects of Dietary Flavonoid Supplementation on the Antioxidant Status of Laying Hens. Brailian Journal of Poultry Science. 2016; 18(4): 663-668.

18. Akbarian A, Michiels J, Degroote J, Majdeddin M, Golian A, De Smet S. Association between heat stress and oxidative stress in poultry; mitochondrial dysfunction and dietary interventions with phytochemicals. J Anim Sci Biotechnol. 2016; 28:7-37

19. Firat O, Cogun H, Yuzereroglu T, Gok G, Kargin F, Kotemen Y. A comparative study on the effects of a pesticide (cypermethrin) and two metals (copper, lead) to serum biochemistry of Nile tilapia, Oreochromis niloticus. Fish Physiology and Biochemistry. 2011; 37:657-666.

20. Bashandy SAE, El Adan SA, Ebarid H, Alhazza IM. Antioxidant Potential of Spirulina platensis Mitigates Oxidative Stress and Reprotoxicity Induced by Sodium Arsenite in Male Rats. Oxidative Medicine and Cellular Longevity. 2016; 2016:1-8.

21. Keshavamurthy SR, Shiva Kumar Manohar CB, Sharadamma KC. Effect Of Antioxidant Formulation Supplementation Through Water on Antioxidant Status of Broiler Chicken. International Journal of Advanced Biological Research. 2013; 3(3):470-474.

22. Pan J, Yang Y, Yang B, et al. Artificial Polychromatic Light Affects Growth and Physiology in Chicks. PloS One. 2014;9: e113595. 
23. Yang Y, Jin SF, Zhong ZT, et al. 2015. Growth responses of broiler chickens to different periods of artificial light. J Anim Sci. 2015; 2: 767-775.

24. Nelson RJ, Blom JMC. Photoperiodic effects on tumor development and immune function. J. Biol. Rhythms.1994; 9:233-249.

25. Yoshioka T, Kawada K, Shimada T, Mori M. Lipid peroxidation in maternal and cord blood and protective mechanism aganist actived oxygen toxicity in the blood. Am J Obstet Gynecol. 1979;135: 372-376.

26. Dowdy S, Wearden S. Statistics for Research. John Wiley\&Sons Press, New York; 1981.

27. Dawson B, Trapp RG. Basic\&Clinical Biostatistics. 3rd ed. Lange Medical Books / McGraw International Editions, New York; 2001.

28. El Halawani, ME. Light spectrum requirement for maximizing breeder hen turkey egg production. Gobbles. 2013; 70(4):6-8.

29. Huber-Eicher B, Suter A,. Spring-Stahli P. Effects of colored light-emitting diode illumination on behavior and performance of laying hens. Poultry Science. 2013; 92:869-873.

30. Halliwell BH, Gutteridge JMC. Free Radicals in Biology and Medicine. 4th ed. Oxford University Press, Oxford; 2007.

31. Nur G, Deveci HA. Histopathological and biochemical responses to the oxidative stress induced by glyphosate-based herbicides in the rainbow trout (Oncorhynchus mykiss). J Cell Neurosci Oxid Stress. 2018; 10(1):656-665.

32. Bowlby GMS. Some preliminary investigations into the effect of light on broilers. World's Poult. Sci. J. 1957;13:214-226.

33. Halevy O, Biran I, Rozenboim I. Various light source treatments affect body and skeletal muscle growth by affecting skeletal muscle satellite cell proliferation in broilers. Comp. Physiol. Biochem. 1998; 120:317-323.

34. Woodard AE, Moore JA, WilsonWO. Effect of wave length of light on growth and reproduction in Japanese quail (Coturnix coturnix japonica). Poult Sci. $1969 ; 48: 118-123$. 